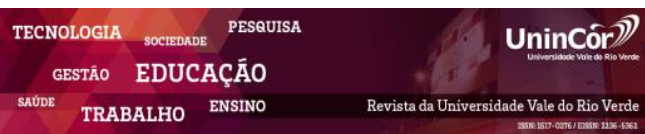

Revista da Universidade Vale do Rio Verde ISSN: 1517-0276 / EISSN: 2236-5362 v. $16\left|\mathbf{n}^{\circ} .3\right|$ Ano 2018

Thuanny Fernandes Brito Noguchi Universidade Vale do Rio Verde Email: enf.thuanny@gmail.com

Elaine Aparecida Rocha Domingues Doutora e Ciências da Saúde. Coordenadora e Docente do Curso de Enfermagem da Universidade Vale do Rio Verde Email: elainerocha.contato@gmail.com

Maiúme Roana Ferreira de Carvalho Docente do Curso de Enfermagem da Universidade Vale do Rio Verde Email: enf maiume@yahoo.com.br

Eliana Alcantra

Doutora professora nos cursos Mestrado Sustentabilidade em Recursos Hídricos e Agronomia da Universidade Vale do Rio Verde. Email: prof.eliana.alcantra@unincor.edu.br

\section{PREVALÊNCIA DE SÍFILIS EM GESTANTE EM UMA CIDADE DO SUL DE MINAS GERAIS: ANÁLISE DOCUMENTAL}

\title{
RESUMO
}

Objetivou relatar a prevalência de sífilis em gestantes no período de janeiro de 2013 a agosto de 2017 em uma cidade do sul de Minas Gerias e identificar o perfil epidemiológico das mães luéticas. Estudo quantitativo, retrospectivo, descritivo, fundamentado em uma análise documental. Verificou se a prevalência de 16 casos/para 1000 nascidos vivos, média de idade materna de 24 anos, 54\% eram pardas, 31\% possui ensino fundamental incompleto, $71 \%$ não tiveram sua ocupação declarada, $35 \%$ apresentaram diagnóstico durante o primeiro trimestre e $35 \%$ durante o segundo, $51 \%$ sífilis primaria, $93 \%$ obteve resultado reagente ao exame não treponêmico durante o pré-natal, $82,5 \%$ foram tratadas e $69 \%$ dos companheiros não foram tratados. Conclui-se que no município há alta prevalência de sífilis em gestantes, as mulheres notificadas portadores da doença possuem uma questão socioeconômica baixa, com tratamento adequado, porém a maioria dos parceiros não foram tratados, o que torna grande a probabilidade de reinfecção.

Palavras-chave: Sífilis. Gravidez. Prevalência.

\section{PREVALENCE OF SYPHILIS IN PREGNANT IN A CITY OF THE SOUTH OF MINAS GERAIS: DOCUMENTARY ANALYSIS}

\begin{abstract}
The objective of this study was to report the prevalence of syphilis in pregnant women from January 2013 to August 2017 in a city in the southern state of Minas Gerais, and to identify the epidemiological profile of the luéticas mothers. Quantitative, retrospective, descriptive study, based on a documentary analysis. The prevalence of 16 cases / 1000 live births, mean maternal age of 24 years, $54 \%$ were brown, $31 \%$ had incomplete elementary education, $71 \%$ had not declared their occupation, $35 \%$ had a diagnosis during the first trimester and $35 \%$ during the second, $51 \%$ primary syphilis, 93\% had a non-treponemal test result during prenatal care, $82.5 \%$ were treated and $69 \%$ of the companions were not treated. It is concluded that in the municipality there is a high prevalence of syphilis in pregnant women, the women notified with the disease have a low socioeconomic issue, with adequate treatment, but most of the partners were not treated, which makes great of reinfection.
\end{abstract}

Keywords: Syphilis. Pregnancy. Prevalence.

Recebido em: 27/08/2018 - Aprovado em: 31/10/2018 - Disponibilizado em: 30/12/2018 


\section{INTRODUÇÃO}

A sífilis é uma doença com impacto na saúde pública, portanto, atualmente vem sendo discutida e estudada por grandes Conselhos de Saúde, que visam buscar possíveis soluções, através de ações especificas realizadas por profissionais enfermeiros por meio de protocolos do Ministério da saúde, conferindo mais autonomia na abordagem da doença (COFEN, 2017a).

Referente a essa morbidade pode se citar duas possíveis teorias, que tentam explicar como foi a disseminação desta. $\mathrm{Na}$ primeira, chamada de colombiana, a sífilis seria endêmica no Novo Mundo trazido por marinheiros espanhóis que participaram da descoberta da américa até a Europa. Outra teoria seria que a sífilis é resultado de mutações e adaptações sofridas por espécies de Treponemas endêmicas do continente africano (AVELLEIRA; BOTTINO, 2006). As Infecções sexualmente transmissíveis fazem parte de um grande problema de saúde pública, gerando problemas sociais, econômicos e sanitários de grande repercussão às populações, especialmente entre mulheres e crianças desde o século passado (MAGALHÃES et al., 2013).
Dentre as Infecções Sexualmente Transmissíveis (IST), a sífilis é uma doença sistêmica causada pela espiroqueta Treponema pallidum. O período de incubação da doença é de 10 a 90 dias e uma média de 21 dias. Ela é transmitida principalmente durante as relações sexuais e também, por vezes, por contato com lesões ou transfusão de sangue, onde o doador está em fase precoce da doença (MINISTÉRIO DA SAÚDE, 2014).

O mesmo autor diz, que os sinais característicos da pessoa afetada são difíceis de reconhecer, porque a lesão inicial que é o cancro, pode ocorrer em áreas menos visíveis. O curso da doença é dividido em quatro fases: primária, secundária, latente e terciária, podendo uma mulher gestante sem tratamento passar para seu feto, levando-o a contrair sífilis congênita.

Todas as pessoas sexualmente ativas devem realizar o teste para diagnosticar a sífilis, principalmente as gestantes, pois a sífilis congênita pode causar aborto, má formação do feto e/ou morte ao nascer. O teste deve ser feito na $1^{\mathrm{a}}$ consulta do prénatal, no $3^{\circ}$ trimestre da gestação e no momento do parto (independentemente de exames anteriores). O cuidado também deve ser especial durante o parto para evitar 
sequelas no bebê, como cegueira, surdez e deficiência mental (MINISTÉRIO da SAÚDE, 2016).

De acordo com o Guia de Vigilância em Saúde (2017) a sífilis congênita é o resultado da disseminação hematogênica do T. pallidum da gestante infectada não tratada ou inadequadamente tratada para o embrião ou feto, por via transplacentária (transmissão vertical). A infecção do embrião pode ocorrer em qualquer fase gestacional ou estágio da doença materna. Os principais fatores que determinam a probabilidade de transmissão são o estágio da sífilis na mãe e a duração da exposição do feto no útero. Portanto, a transmissão será maior nas fases iniciais da doença, quando há mais espiroquetas na circulação. A taxa de transmissão é de 70-100\% nas fases primária e secundária, $40 \%$ na fase latente recente e $10 \%$ na latente tardia.

Segundo Brasil (2016), a notificação compulsória de gestante com sífilis em todo o território nacional foi instituída por meio da Portaria no 33 de 14 de julho de 2005.

Segundo Brasil - Departamento de informática do SUS (2016), no período de 2005 a junho de 2016 foram notificados no SINAN - Sistema de Informação de agravos e notificações um total de 169.546 casos de sífilis em gestantes dos quais $44,8 \%$ eram residentes na região Sudeste, 18,7 na região Nordeste, $18,0 \%$ na região Sul, $10,4 \%$ na região Norte e 7,5\% na região Centro-Oeste.

Conforme o Boletim Epidemiológico de Sífilis (2017) em 2016, o número total de casos notificados no Brasil foi de 37.436, dos quais 17.551(46,9\%) foram casos residentes na região Sudeste, 6.571(17,6\%) no Nordeste, $6.608(17,7 \%)$ no Sul, $11,9 \%$ no Norte e 9,8\% no Centro-Oeste.

\section{Segundo o Protocolo Clínico e} Diretrizes Terapêuticas (2015) quando se fala de tratamento da sífilis, a penicilina é o principal medicamento de escolha, sendo que níveis de penicilina superiores a 0,018 mg por litro são considerados suficientes e devem ser mantidos por pelo menos sete a 10 dias na sífilis recente, e por duração mais longa na sífilis tardia, para gestantes com alergia confirmada à penicilina: como não há garantia de que outros medicamentos consigam tratar a gestante e o feto, impõe-se a dessensibilização e o tratamento com Penicilina Benzatina. Na impossibilidade de realizar a dessensibilização durante a gestação, a gestante deverá ser tratada com Ceftriaxona de $1 \mathrm{~g}$ a $2 \mathrm{~g}$ por no mínimo 8 dias. Guinsburg, Santos (2010), fala que o 
tratamento da sífilis em gestante com penicilina no primeiro trimestre costuma evitar a infecção fetal. Após esta fase, trata também.

Diante o grande problema que a Sífilis se tornou, o Ministério da Saúde junto ao Conselho Federal de Enfermagem lançou em 2017 uma nova estratégia que, por meio de uma resolução, possibilita a aplicação de Penicilina nas unidades de atenção básica pela equipe de enfermagem, mediante prescrição médica ou de enfermagem. A resolução ainda afirma que a ausência do médico na unidade não impossibilita a aplicação da medicação pela equipe de enfermagem (COFEN, 2017)

Mediante esse cenário, que apresenta a alta incidência de sífilis materna e congênita na região sudeste e o grande problema de saúde pública, verifica-se a necessidade de realizar pesquisas no que refere a temática, principalmente na cidade de Três Corações, MG uma vez que existem vários casos de gestantes luéticas.

Portanto, o objetivo do estudo foi relatar a prevalência de sífilis em gestantes e identificar o perfil epidemiológico das mães luéticas no município de Três CoraçõesMinas Gerais.

\section{MATERIAL E MÉTODOS}

O estudo trata-se de uma pesquisa descritiva, retrospectiva, com abordagem quantitativa.

O estudo foi realizado em Três Corações, localizado no sul de Minas Gerais, no Serviço de Vigilância Epidemiológica do município. Participaram do estudo gestantes diagnosticadas e notificadas, residentes em Três Corações, entre janeiro de 2013 a agosto de 2017.

Os dados foram coletados a partir das fichas de notificação do SINAN, obtidas junto ao Serviço de Vigilância Epidemiológica da Secretaria de Saúde de Três Corações. Foi realizado um levantamento das variáveis sociodemográficos das gestantes, como: faixa etária, raça/cor, escolaridade e ocupação.

As variáveis clínicas estudadas foram: realização do pré-natal, momento do diagnóstico da sífilis, adequabilidade do tratamento e tratamento do parceiro simultâneo à gestante.

Os dados foram armazenados e tabulados em uma planilha eletrônica utilizando o programa Excel 365 
$\left(\right.$ Microsoft $\left.^{\circledR}\right)$ e os resultados apresentados em figura e tabelas.

O estudo obedeceu aos princípios da Resolução 466/12 do Conselho Nacional de Saúde e foi aprovado pelo Comitê de Ética em Pesquisa da Universidade Vale do Rio Verde - Unincor, com o parecer $n^{\circ}$ 2.332.848.

\section{RESULTADOS E DISCUSSÃO}

No período de 2013 a 2017 foram diagnosticados e verificados 80 casos de sífilis em gestantes no município de Três Corações, sendo que entre 2013 e 2014 o número de casos aumentou substancialmente, passando de 10 para 23 casos (Figura1).

Figura 1 - Número de casos notificados de sífilis em gestantes em Três Corações, MG, 2013-2017. (n=80)

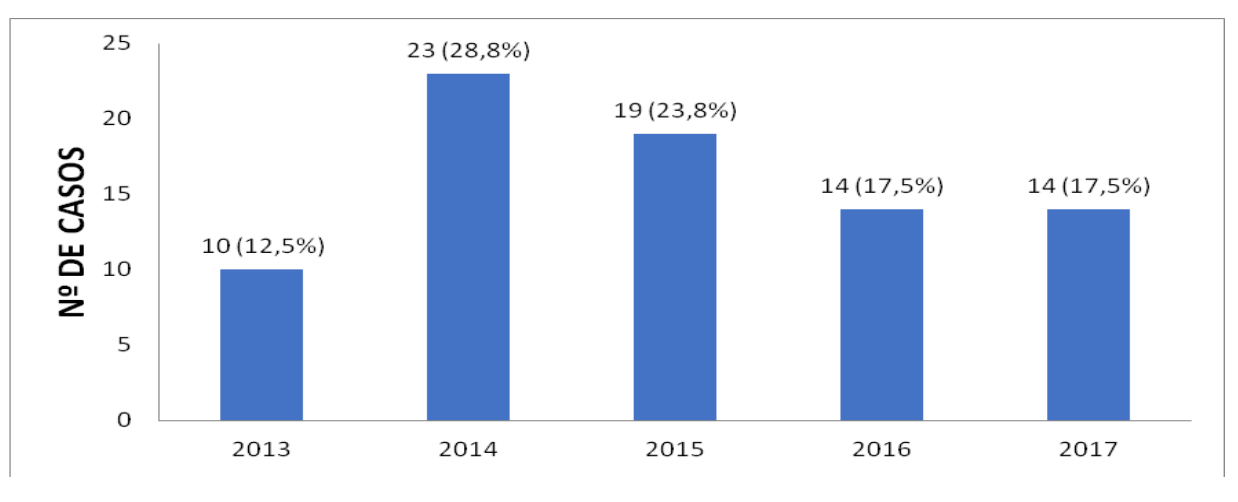

Fonte: Autores.

Em 2015 no Brasil, houve 3.165.873 partos de nascidos vivos e uma margem de 33.365 notificações de sífilis em gestantes, diante esses dados o Brasil possui uma taxa de prevalência de 10,5 casos de sífilis em gestantes/1.000 nascidos vivos, taxa superada pela região Sudeste (12,5 casos de sífilis em gestantes/1.000 nascidos vivos), em 2016 a prevalência em Minas Gerais foi de 10,04 casos de sífilis em gestantes/1.000 nascidos vivos, mantendo o nível nacional. (BOLETIM EPIDEMIÓLOGICO, 2016).

Entre janeiro de 2013 a outubro de 2017 ocorreram um total de 4,983 partos nascidos vivos, em Três Corações, totalizando assim uma prevalência de 16,05 casos de sífilis em gestante/1.000 nascidos vivos (80 casos), nos referidos anos (Figura 2). 
Figura 2 - Prevalência de Sífilis em Gestante a cada 1000 nascidos vivos no período de 2013-2017, no município de Três Corações, MG

\section{Prevalência de Sifílis em Gestantes}

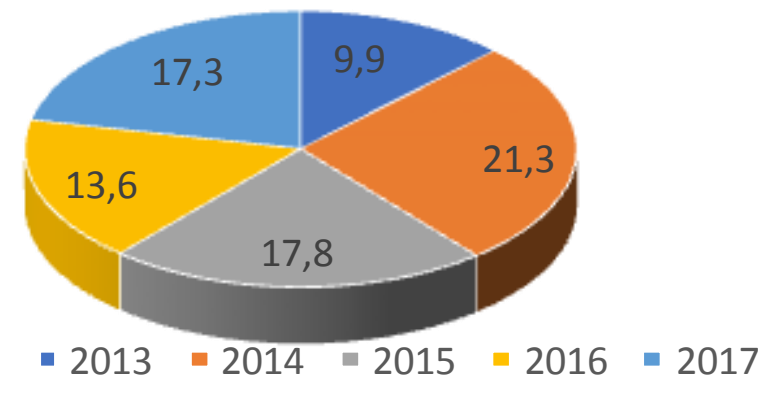

Fonte: Autores.

Em relação à variável idade materna, a idade mínima encontrada foi de 14 anos e máxima de 43 anos, com média de 23,98 anos. Observa-se uma predominância na faixa etária de 14 a 20 anos, correspondendo a 34 casos, $43 \%$ do total de casos do período estudado, seguida por $38 \%$ na faixa de 21 a 30 anos, correspondendo a 30 casos. A maioria das mulheres eram pardas 43(54\%), com ensino fundamental incompleto 25(31\%). A principal ocupação foi dona de casa $14(18 \%)$. Destaca-se que, em relação à ocupação, uma elevada porcentagem desconhecia essa informação 57 (71\%) (Tabela 1). 
Tabela 1- Distribuição das características sociodemográficos maternas dos casos notificados de sífilis em gestantes em Três Corações, MG, 2013-2017. (n=80)

\begin{tabular}{lcc}
\hline Características & N & \% \\
\hline Faixa etária(anos) & 34 & 43 \\
$14-20$ & 30 & 38 \\
$21-30$ & 14 & 18 \\
$31-40$ & 02 & 3 \\
$>40$ & & \\
Cor da pele & 14 & 18 \\
Branca & 19 & 24 \\
Preta & 02 & 3 \\
Amarela & 43 & 54 \\
Parda & 02 & 3 \\
Ignorada & & \\
Escolaridade & 25 & 31 \\
Fundamental incompleto & 12 & 15 \\
Fundamental Completo & 15 & 19 \\
Ensino Médio Incompleto & 23 & 29 \\
Ensino Médio Completo & 05 & 6 \\
Ignorado & & 4 \\
Ocupação & 03 & 18 \\
Estudante & 14 & 3 \\
Dona de casa & 02 & 4 \\
Doméstica & 03 & 1 \\
Manicure & 01 & 71 \\
Babá & 57 & \\
Não informado & & \\
\hline
\end{tabular}

Fonte: Autores.

Quanto às características clínicas das gestantes (Tabela 2), verificou-se que a idade gestacional do diagnóstico da sífilis foi realizada igualmente durante o primeiro e segundo trimestre gestacional, com $35 \%$ dos casos. Em relação a classificação da doença predominou a primária $51 \%$, seguida da latente $35 \%$. Os resultados referentes ao teste não treponêmico realizado durante o prénatal, obteve predominância o reagente $93 \%$. No que se refere ao tratamento da sífilis, $82,5 \%$ foram tratadas adequadamente e o tratamento foi com Penicilina G Cristalina 2. 400.000 UI. Quanto ao tratamento do parceiro $69 \%$ não 0 realizaram concomitantemente à gestante.

Tabela 2 - Distribuição das características clínicas dos casos notificados de sífilis em gestantes em Três Corações, MG, 2013-2017. ( $\mathrm{n}=80)$

\begin{tabular}{llc}
\multicolumn{1}{c}{ Características da assistência pré-natal } & $\mathbf{N}$ & \% \\
\hline Idade gestacional & & 35 \\
Primeiro Trimestre & 28 & 35 \\
Segundo Trimestre & 28 & 28 \\
Terceiro Trimestre & 22 & 3 \\
Ignorado & 02 &
\end{tabular}

\section{Classificação clínica}




\begin{tabular}{lcc} 
Primária & 41 & 51 \\
Secundária & 05 & 6 \\
Latente & 28 & 35 \\
Não informado & 06 & 7,5 \\
Teste não treponêmico & & 93 \\
Reagente & 74 & 1 \\
Não reagente & 01 & 3 \\
Não realizado & 02 & 4 \\
Ignorado & 03 & 72,50 \\
Tratamento das gestantes & & 16,25 \\
Adequado & 66 & 1,25 \\
Não realizado & 13 & 29 \\
Ignorado & 01 & 69 \\
Tratamento do parceiro das gestantes & & 3 \\
Sim & 23 & \\
Não & 55 & 02 \\
Ignorado & & \\
\hline
\end{tabular}

Fonte: Autores.

A cobertura de assistência pré-natal no Brasil é praticamente universal e alcança valores superiores a 90,0\%, independentemente da região geográfica do País ou características maternas (DOMINGUES et al., 2014).

Domingues et al. (2014), coloca ainda em seu estudo que a região do Brasil que mais ocorre sífilis em Gestante é a região Sudeste.

Os resultados deste estudo indicam que a prevalência de Sífilis em Três Corações apresentou um crescimento no período de 2013 para 2014. Este aumento também foi enfatizado em outras cidades brasileiras (LIMA et al., 2013; COSTA et al., 2013; BONI, PAGLIARI, 2016)

Pode observar que a prevalência encontrada em 2017 no município é bem maior que a encontrada na região sudeste e a nível nacional, o que torna preocupante a situação do município (DATASUS, 2017).

Referente a idade materna de maior prevalência, pode-se observar uma semelhança a outras pesquisas publicadas (CAMPOS et al., 2010; BONI, PAGLIARI, 2016; NONATO, MELO, GUIMARÃES, 2015).

Conforme as pesquisas de Domingues, (2014); Bordignon, (2017) verifica-se uma diferenciação de idades, visto que em seus estudos as idades encontradas foram superiores as encontradas nesta pesquisa.

Todos os autores citados a cima mostram que a maioria das mulheres luéticas são pardas e possuem baixa escolaridade, o que remete a realidade encontrada nessa pesquisa. A baixa escolaridade está relacionada ao menor acesso à informação, a um limitado entendimento da importância 
dos cuidados com a saúde e, principalmente, às medidas de prevenção da infecção (NONATO, MELO e GUIMARÃES, 2015).

No que se refere a ocupação das gestantes com Sífilis o presente estudo verificou a maior porcentagem de dado tido como não informado, o que traz uma indagação e uma reflexão sobre a atuação do enfermeiro frente a consulta de enfermagem e o preenchimento correto da ficha de notificação, visto que são dados essenciais para o levantamento do perfil do município.

Estudos mostram que a infecção vertical (mãe - feto) pode ocorrer em qualquer fase gestacional ou estágio da doença materna. Os principais fatores que determinam a probabilidade de transmissão são o estágio da sífilis na mãe e a duração da exposição do feto no útero. Portanto, a transmissão será maior nas fases iniciais da doença, quando há mais espiroquetas na circulação. A taxa de transmissão é de 70$100 \%$ nas fases primária e secundária, $40 \%$ na fase latente recente e $10 \%$ na latente tardia (GUIA DE VIGILÂNCIA EM SAÚDE, 2017).

No presente estudo verificou-se que as gestantes tiveram o diagnóstico no primeiro e segundo trimestre na mesma proporção numérica 35 (28\%). Conforme o Boletim Epidemiológico (2017) quando se avalia a idade gestacional de detecção da sífilis em gestantes, obtém-se um resultado, em 2016, que a maior proporção das mulheres $(37,0 \%)$ foi diagnosticada no primeiro trimestre. Em comparação ao ano anterior, isso representa um aumento de $15,1 \%$, demonstrando uma ampliação do diagnóstico precoce durante o pré-natal. Os diagnósticos realizados no segundo trimestre representaram $29,0 \%$.

No presente estudo verificou-se que 41\%(51) das gestantes apresentavam a Sífilis primaria, conforme Damasceno et al (2014), a sífilis primária ocorre após três semanas de exposição; há o aparecimento de úlcera, única, medindo entre 0,3 e 3,0 cm, indolor, no local da inoculação, com resolução espontânea em três a seis semanas, podendo ocasionalmente aparecer lesões múltiplas, sendo mais comuns quando associadas à coinfecção pelo HIV. Porém o Boletim epidemiológico de Sífilis (2017) verifica em análise de resultados das fichas de notificação de Sífilis em Gestante no Brasil, que a maioria dos diagnósticos da apresentação da doença são realizados de maneira inadequada, o que infelizmente interfere em dados fidedignos referente a classificação da doença. Diante esse cenário é imprescindível que o profissional que realiza o diagnóstico e conclui a classificação deve ter conhecimento especifico, com uma atuação coerente, visto que o tratamento correto está interligado a classificação da 
doença, o que pode interferir na cura da doença.

No que diz respeito a realização do Teste não Treponêmico durante o pré-natal, esse resultado não difere dos identificados em outros estudos, pois esses achados levantam uma preocupação a respeito da doença, uma vez que, quanto mais precoce for o diagnóstico e o tratamento, menor será o tempo de exposição do feto ao Treponema pallidum.

O Ministério da Saúde preconiza a realização de testes treponêmico e não treponêmico durante o primeiro e o terceiro trimestre da gestação, e no momento do parto. A gestante infectada pelo T. pallidum vai apresentar os testes treponêmicos reagentes indefinidamente, mesmo que já tratada (BRASIL, 2015; BRASIL, 2014; FRANSCISCO, 2014). Os não treponêmicos tendem à negativação após o tratamento. No entanto, podem apresentar testes não treponêmicos persistentemente reagentes, em baixas titulações, após o tratamento. Por isso, na ausência de tratamento, gestantes com títulos baixos em testes não treponêmicos devem ser tratadas. Nesse estudo pode observar que o teste treponêmico no pré-natal não foi realizado em nenhuma gestante notificada, o que torna preocupante o diagnostico final, já que a possibilidade de existirem falsos-positivos ou falsos negativos de teste não treponêmico é elevado.
Segundo o guia de bolso de Sífilis (2016), toda gestante diagnosticada com sífilis, deverá ter o seu tratamento iniciado o mais precocemente possível, mesmo se ainda persistirem dúvidas sobre o diagnóstico. Para as gestantes a única forma efetiva de tratamento, para evitar a sífilis congênita é o uso de Penicilina Benzatina, com dose diferenciada para cada fase da infecção. Normalmente quando se trata de sífilis primária o tratamento é feito com duas series de 2.400.000 U cada uma de Penicilina Benzatina, obtendo um espaço de tempo de 1 semana. Mediante esse tratamento possivelmente a paciente estará curada, porém deve se fazer acompanhamento sorológico por meio de exames laboratoriais para confirmação da cura.

Campos et al. (2010), traz que quando a sífilis nas gestantes não é tratada, ou é inadequadamente tratada, pode ocasionar abortamentos, prematuridade e natimortalidade. Quando ocorre a infecção do concepto a partir de mães não tratadas, em aproximadamente $40 \%$ dos casos acontecem aborto espontâneo, natimorto ou óbito perinatal.

\section{Referente a isso o Boletim} Epidemiológico de Sífilis (2017), traz que observadas as informações de gestantes não tratadas, por estado, registraram-se proporções de $1,0 \%$ a $9,5 \%$. Os estados com as menores proporções foram: Sergipe 
$(1,0 \%)$, Rio de Janeiro (2,0\%), Acre (2,2\%), Amazonas (2,2\%), Mato Grosso (2,2\%) e Tocantins (2,4\%). Já os estados com os piores resultados foram: Rondônia $(9,5 \%)$, Minas Gerais (8,6\%), Espírito Santo (8,3\%) e Paraná $(8,2 \%)$. O que torna preocupante a situação de Minas Gerais.

Segundo o estudo de Magalhães et al, (2013), o principal fator de falha no tratamento da gestante foi a falta e/ou a inadequação do tratamento do parceiro, fato que se assemelha ao presente estudo, visto que cerca de 55(69\%) dos parceiros não realizaram tratamento, o que dificulta a cura da gestante e aumenta a probabilidade de sífilis congênita. Esses resultados são encontrados em vários estudos, que vem em contrapartida confirmar os resultados deste (LORANZI, MADI,2001; DONALÍSIO, FREIRE， MENDES， 2007; FIGUEIRÓFILHO, et al. 2007; TREVISAN, et al. 2002).

Diante do exposto destaca-se a importância da inclusão do parceiro no prénatal, pois essa estratégia de abordagem do problema é determinante para a cura eficaz da mãe e, consequentemente, para o fim do agravo.

Este trabalho apresentou algumas limitações para seu desenvolvimento, condicionados à qualidade dos registros. Evidenciou -se um número elevado de dados faltantes, ou preenchidos incorretamente, possível reflexo da ausência do registro pelo profissional no qual realizou a notificação.

Os fatores associados encontrados neste estudo sugerem falhas na assistência pré-natal e indicam a necessidade de propor novas estratégias com o objetivo de reduzir a possível transmissão vertical da sífilis, a exemplo da capacitação continuada dos profissionais, via discussão dos casos, fortalecimento da vigilância epidemiológica, para o monitoramento dos resultados do VDRL de gestantes no sistema de laboratórios, e abordagens integradas de prevenção da sífilis e do HIV/aids, dando mais visibilidade à sífilis congênita, todavia um problema de Saúde Pública longe de ser eliminado.

\section{CONCLUSÃO}

Conclui-se que os achados deste estudo mostram uma alta prevalência de Sífilis em gestantes no município $(16,05$ casos de sífilis em gestante/1.000 nascidos vivos), o que se torna preocupante, visto que o município ainda possui uma grande taxa de subnotificações. Nota-se que as mulheres notificadas portadores da doença possuem uma questão socioeconômica baixa, o que pode ser correlacionado a falta de informação e conhecimento sobre os riscos causados pela doença. A maioria das mulheres foram tratadas adequadamente, porém a maioria dos 
parceiros não foram tratados, o que torna grande a probabilidade de reinfecção e possível infecção vertical, levando a sífilis congênita

Verifica-se também, alguns pontos frágeis da notificação, verificação de casos, assistência e prevenção da sífilis, visto que durante a coleta de dados foi observado um preenchimento incorreto, sem fundamentação de algumas informações contidas nas fichas. A redução da ocorrência da sífilis no período gestacional e, consequentemente, da sífilis congênita, somente será possível quando a adoção de medidas mais efetivas de prevenção e controle forem sistematicamente aplicadas, os profissionais da saúde, com prioridade o enfermeiro, devem participar ativamente na realização dessas ações preventivas inclusive ao de acompanhamento e tratamento desta enfermidade, buscando atuar também na parte epidemiológica com o preenchimento adequado da notificação , tornando assim a informação fidedigna e somente assim o perfil epidemiológico do município poderá ser adequadamente traçado.

\section{REFERÊNCIAS}

AVELLEIRA, J. C. R.; BOTTINO, G. Sífilis: diagnóstico, tratamento e controle. Anais

Brasileiro de Dermatologia, v. 81, n. 2, p. 111126, 2006. Disponível em: < http://www.scielo.br/pdf/abd/v81n2/v81n02a02.p df > Acesso em: Março 2017.
BONI, S. M.; PAGLIARI, P. B. incidência de Sífilis Congênita e sua prevalência em gestantes em um município do noroeste do paraná. Saúde e Pesquisa, v. 9, n. 3, p. 517-524, 2016. Disponível em: <

http://periodicos.unicesumar.edu.br/index.php/sa udpesq/article/view/5530/2919 > Acesso em: outubro de 2017

BORDIGNON, R. P.; CARDOSO, K.;

BARRETO, C. N. Sífilis na Gestação e Sífilis Congênita: Os desafios para Saúde Pública.

Revista dos TCC's e Semana Acadêmica da ULBRA Cachoeira do Sul, v. 1, n. 1, 2017.

Disponível em: <

http://www.ulbracds.com.br/index.php/rtccsa/arti cle/view/1029/0 > Acesso em: outubro de 2017.

BRASIL. Ministério da Saúde. Boletim

Epidemiológico- sífilis 2016, v. 47, n. 35, p. 144, 2016. Disponível em: < http://www.aids.gov.br/pt-br/pub/2016/boletimepidemiologico-de-sifilis-2016 > Acesso em: junho 2017.

Ministério da Saúde. Sistema de Informação de Agravos de Notificação - Sinan Net, Sífilis Congênita, 2016. Disponível em : < http://portalsinan.saude.gov.br/sifilis-congenita > Acesso em: Março de 2017

. Ministério da Saúde.

\section{DATASUS Tecnologia da Informação a}

Serviço, 2017. Disponível em: < http://tabnet.datasus.gov.br/cgi/deftohtm.exe?sin annet/cnv/sifilisgestanteMG.def $>$ Acesso em: Março de 2017.

Ministério da Saúde. DATASUS Tecnologia da Informação a Serviço, 2017. Disponível em: < http://vigilancia.saude.mg.gov.br/index.php/sistemasde-informacao/nascidos-vivos-sinasc/ > Acesso em: Dezembro de 2017.

Ministério da Saúde. Boletim

Epidemiológico- sífilis 2017, v. 48, n. 36, p. 144, 2017. Disponível em: < http://www.aids.gov.br/es/node/65020 > Acesso em: outubro 2017.

Ministério da Saúde. Protocolo Clinico

e Diretrizes Terapêuticas, Brasilia, p. 1-120,

2015. Disponível em: <

http://www.aids.gov.br/pt-

br/pub/2015/protocolo-clinico-e-diretrizes- 
terapeuticas-para-atencao-integral-pessoas-cominfeccoes > Acesso em: agosto de 2017

Ministério da Saúde.

DATASUS Tecnologia da Informação a

Serviço, 2017. Disponível em: <

http://tabnet.datasus.gov.br/cgi/deftohtm.exe?sin

asc/cnv/nvmg.def > Acesso em: outubro de 2017.

. Ministério da Saúde. Secretaria de

Ciência, Tecnologia e Insumos Estratégicos.

Protocolo Clínico e Diretrizes Terapêuticas

Infecções Sexualmente Transmissíveis.

Brasília, v. 20, n. 2, p. 225-233, 2015. Disponível em: <

http://conitec.gov.br/images/Consultas/Relatorio s/2015/Relatorio_PCDT_IST_CP.pdf > Acesso em: outubro de2017.

Ministério da Saúde (BR). Secretaria de Vigilância em Saúde. Guia de Vigilância em Saúde, Secretaria de Vigilância em Saúde. Brasília, v. 43, n. 4, p. 812, 2014. Disponível em: $<$ file:///C:/Users/Thuanny/Downloads/guiavigilancia-saude-final-2.pdf > Acesso em: outubro 2017.

Ministério da Saúde (BR). Secretaria de Vigilância em Saúde. Guia de Bolso para o Manejo de Sífilis em Gestante e Sífilis Congênita. São Paulo p. 1- 112, 2016.

Disponível em: <

http://www.saude.campinas.sp.gov.br/doencas/si filis/guiadebolsodasifilis 2edicao2016.pdf > Acesso em: outubro 2017.

. Ministério da Saúde. Secretaria de Vigilância em Saúde. Guia de Vigilância em Saúde. Brasilia-DF p. 1-222, 2017. Disponível em: <

http://www.hc.ufu.br/sites/default/files/tmp//volu me_2_guia_de_vigilancia_em_saude_2017.pdf $>$ Acesso em: outubro 2017.

CAMPOS, A. L. de A.; et al. Epidemiologia da Sífilis Gestacional em Fortaleza, Ceará, Brasil: um agravo sem controle. Caderneta de Saúde Pública, Rio de Janeiro, v. 26, n. 9, p. 17471755, set- 2010. Disponível em: < file:///C:/Users/Thuanny/Downloads/08\%20(2).p df > Acesso em: outubro de 2017
COFEN - Conselho Federal de Enfermagem. Cofen apresenta nota técnica em audiência sobre a sífilis congênita. Ascom-Cofen, 2017a. Disponível em: < http://www.cofen.gov.br/cofen-apresenta-notatecnica-em-audiencia-sobre-sifiliscongenita_52224.html > Acesso em: Maio de 2017

COFEN - Conselho Federal de Enfermagem. Cofen apresenta nota técnica sobre administração de Penicilina Benzatina. AscomCofen, 2017b. Disponível em: < http://www.cofen.gov.br/cofen-publica-notatecnica-sobre-administracao-da-penicilinabenzatina 52797.html > Acesso em: setembro de 2017

COSTA, C. da C. et al. Sífilis Congênita no Ceará: analise epidemiológica de uma década. Revista Escola de Enfermagem da Usp, v. 47, n. 1, p. 152-159, 2013. Disponível em: < https://pdfs.semanticscholar.org/af34/a3cb44aa3 abacb8e752db89083cc09d890fb.pdf $>$ Acesso em: outubro de 2017.

DAMASCENO, A.B. A.; et al. Sífilis na Gravidez. Revista HUPE, Rio de Janeiro, v. 13, n. 3, p. 88-94, 2014. Disponível em: < file:///C:/Users/Thuanny/Downloads/v13n3a14\% 20(4).pdf > Acesso em: outubro de 2017.

DONALÍSIO, M. R.; FREIRE, J. B.; MENDES, E. T. Investigação da sífilis congênita na microrregião de Sumaré, Estado de São Paulo, Brasil - desvelando a fragilidade do cuidado à mulher gestante e ao recém-nascido.

Epidemiologia e Serviços de Saúde, v. 16, n. 3, p. 165-173, 2007. Disponível em: < http://scielo.iec.pa.gov.br/pdf/ess/v16n3/v16n3a0 3.pdf > Acesso em: outubro de 2017

DOMINGUES, R. M. S. M.; et al. Prevalência de Sífilis na Gestação e testagem pré-natal: Estudo nascer do Brasil. Revista de Saúde Pública, v.48, n. 5, p. 766-774, 2014. Disponível em: <

http://www.redalyc.org/html/672/67237027007/ > Acesso em: outubro de 2017.

FIGUEIRÓ-FILHO, E. A.; et al. Sífilis congênita como fator de assistência pré-natal no município de Campo Grande - MS. DST Jornal Brasileiro de Doenças Sexualmente Transmissíveis, v. 
19, n. 3, p. 139-143, 2017. Disponível em: < http://www.dst.uff.br//revista19-3-2007/5.pdf > Acesso em: outubro de 2017

FRANCISCO, V. C. C. Sífilis Congênita no município de Macapá /AP: análise dos dados registrados no sistema de informação de agravos de notificação (sinan), no período de 2007 a 2012. Universidade Federal do Amapá, 2014. Disponível em: < http://www2.unifap.br/ppcs/files/2014/09/Vivan e-Cristina-Cardoso-Francisco.pdf $>$ Acesso em: outubro de 2017.

GUNSBURG, R.; SANTOS, A. M. N. dos. Critérios de diagnósticos e tratamento da sífilis congênita. Sociedade Brasileira de Pediatria, São Paulo, 2010. Disponível em: < http://www.sbp.com.br/pdfs/tratamento_sifilis.pd f > Acesso em Março de 2017.

LIMA, M. G.; et al. Incidência e fatores de risco para sífilis congênita em Belo Horizonte, Minas Gerais, 2001-2008. Ciências de saúde coletiva [online], v.18, n.2, p.499-506, 2013. Disponível em: < http://www.scielo.br/scielo.php?pid=S1413$\underline{81232013000200021 \& \text { script }=\text { sci_abstract\&tlng= }}$ pt > Acesso em: outubro 2017.

\begin{tabular}{l}
\hline Thuanny Fernandes Brito Noguchi \\
Acadêmica do Curso de Enfermagem da \\
Universidade Vale do Rio Verde. \\
\hline \\
\hline Elaine Aparecida Rocha Domingues \\
Doutora em Ciências da Saúde pela Universidade \\
Estadual de Campinas (UNICAMP). Coordenadora e \\
Docente do Curso de Enfermagem da Universidade \\
Vale do Rio Verde.
\end{tabular}

\section{Maiúme Roana Ferreira de Carvalho}

Mestre em Ciências da Saúde. Docente do Curso de Enfermagem da Universidade Vale do Rio Verde.

\footnotetext{
Eliana Alcantra

Engenheira Agronoma, Mestre e Doutora em Entomologia. Atua como docente nos cursos Mestrado Sustentabilidade em Recursos Hídricos e Agronomia, UninCor.
}

LORENZI, DE D. R. S.; MADI, J. M. Sífilis Congênita como Indicador de Assistência Prénatal. Revista Brasileira de Ginecologia e Obstetrícia, v. 23, n. 10, p. 647-652, 2001. Disponível em: http://www.scielo.br/pdf/rbgo/v23n10/8489.pdf > Acesso em: outubro de 2017

MAGALHÃES, D. M. dos S.; et al. Sífilis Materna e Congênita: ainda um desafio. Caderneta de Saúde Pública, Rio de Janeiro, v. 29, n. 6, p. 1109-1120, jun., 2013. Disponível em: <

http://www.scielo.br/pdf/csp/v29n6/a08v29n6.pd f > Acesso em: Março 2017

NONATO, S. M.; M, A. P. S.; GUIMARÃES, M. D. C. Sífilis na gestação e fatores associados à Sífilis Congênita em Belo Horizonte - MG 2010-2013. Epidemiologia Serviço de Saúde, Brasília, v. 24, n. 4, p. 681-694, out-dez 2015. Disponível em: <

http://scielo.iec.pa.gov.br/pdf/ess/v24n4/v24n4a1 $\underline{0 . p d f}>$ Acesso em: outubro de 2017

TREVISAN. M. R. et al. Perfil da assistência pré-natal entre usuárias do Sistema Único de Saúde em Caxias do Sul. Revista Brasileira de Ginecologia e Obstetrícia, v. 24, n. 5. P. 293299, 2002. Disponível em: < http://www.scielo.br/pdf/rbgo/v24n5/10650.pdf > Acesso em: outubro de 2017 